Iternational Electronic Journal of Algebra

Volume 27 (2020) 263-270

DOI: $10.24330 /$ ieja.663084

\title{
A CONDITION FOR CYCLIC CHIEF FACTORS OF FINITE GROUPS
}

\author{
Changwen Li \\ Received: 20 June 2019; Revised: 3 July 2019; Accepted: 16 July 2019 \\ Communicated by Arturo Magidin

\begin{abstract}
In this paper, we find a condition under which every chief factor
of $G$ below a normal subgroup $H$ of $G$ is cyclic by using the $\tau$-supplemented

subgroups. Some recent results are generalized.
\end{abstract}

Mathematics Subject Classification (2010): 20D10, 20D20

Keywords: Sylow subgroup, $\tau$-supplemented, $p$-nilpotent

\section{Introduction}

In this paper, $G$ always denotes a finite group and $p$ is a prime. A subgroup $H$ of $G$ is called $S$-quasinormal (or $S$-permutable) [6] in $G$ if it permutes with every Sylow subgroup of $G ; H$ is $S$-supplemented [13] in $G$ if there exists a subgroup $T$ of $G$ such that $G=H T$ and $H \cap T \leq H_{s G}$, where $H_{s G}$ is the subgroup of $H$ generated by all those subgroups of $H$ which are $S$-quasinormal in $G$. In [14], Skiba proved the following significant result:

Theorem 1.1. Let $H$ be a normal subgroup of a group $G$. Suppose that for every non-cyclic Sylow subgroup $P$ of $H$, all maximal subgroups of $P$ or all cyclic subgroups of $P$ of prime order and order 4 are $S$-supplemented in $G$. Then every $G$-chief factor below $H$ is cyclic.

A subgroup $H$ of $G$ is $S S$-quasinormal [8] in $G$ if there is a subgroup $B$ of $G$ such that $G=H B$ and $H$ permutes with every Sylow subgroup of $B ; H$ is $S S$ supplemented [17] in $G$ if there is a subgroup $T$ of $G$ such that $G=H T$ and $H \cap T$ is $S S$-quasinormal in $G$. In [17], Yan et al. strengthened Theorem 1.1 as follows:

Theorem 1.2. Let $H$ be a normal subgroup of a group $G$. Suppose that for every non-cyclic Sylow subgroup $P$ of $H$, all maximal subgroups of $P$ or all cyclic subgroups of $P$ of prime order and order 4 are $S S$-supplemented in $G$. Then every $G$-chief factor below $H$ is cyclic.

The project is supported by the Natural Science Foundation of China (No: 11571145). 
A subgroup $H$ of $G$ is said to be $s$-semipermutable [15] in $G$ if $H$ permutes with all Sylow $q$-subgroups of $G$ for the primes $q$ not dividing $|H| ; H$ is $\tau$-quasinormal [11] in $G$ if $H$ permutes with every Sylow subgroup $Q$ of $G$ such that $(|H|,|Q|)=1$ and $\left(|H|,\left|Q^{G}\right|\right) \neq 1 ; H$ is $\tau$-supplemented [10] in $G$ if $G$ has a subgroup $T$ such that $G=H T$ and $H \cap T \leq H_{\tau G}$, where $H_{\tau G}$ is the subgroup generated by all those subgroups of $H$ which are $\tau$-quasinormal in $G$. It is easy to see that if a subgroup $H$ of $G$ is $S S$-supplemented in $G$, then $H$ is $\tau$-supplemented in $G$. However, the converse does not hold in general. In this paper, we get the following result which is an extension of Theorems 1.1 and 1.2.

Theorem 1.3. Let $H$ be a normal subgroup of $G$. Suppose that there exists a normal subgroup $X$ of $G$ such that $F^{*}(H) \leq X \leq H$ and for every non-cyclic Sylow subgroup $P$ of $X$, either all maximal subgroups of $P$ lacking supersolvable supplements in $G$ or all cyclic subgroups of $P$ of prime order and order 4 without supersolvable supplements in $G$ are $\tau$-supplemented in $G$. Then every $G$-chief factor below $H$ is cyclic.

Here $F^{*}(G)$ is the generalized Fitting subgroup of $G$, i.e., the product of all normal quasinilpotent subgroups of $G$ (see [4, X, 13]).

\section{Preliminaries}

Lemma 2.1 ([10, Lemma 2.2]). Let $H$ be a $\tau$-supplemented subgroup of a group $G$.

(1) If $H \leq L \leq G$, then $H$ is $\tau$-supplemented in $L$.

(2) If $N \unlhd G, N \leq H \leq G$ and $H$ is a p-group for some prime $p$, then $H / N$ is $\tau$-supplemented in $G / N$.

(3) If $H$ is a $\pi$-subgroup and $N$ is a normal $\pi^{\prime}$-subgroup of $G$, then $H N / N$ is $\tau$-supplemented in $G / N$.

Lemma 2.2 ([7, Lemma 2.12]). Let $P$ be a Sylow p-subgroup of a group $G$, where $p$ is a prime with $(|G|, p-1)=1$. If every maximal subgroup of $P$ has a $p$-nilpotent supplement in $G$, then $G$ is p-nilpotent.

Lemma 2.3 ([2, A, 1.2]). Let $U, V$, and $W$ be subgroups of a group $G$. Then the following statements are equivalent:

(1) $U \cap V W=(U \cap V)(U \cap W)$;

(2) $U V \cap U W=U(V \cap W)$. 
Lemma 2.4. Let $H$ be a p-subgroup of $G$ and $N$ be a normal subgroup of $G$. If $H$ is $\tau$-quasinormal in $G$, then $H \cap N$ is also $\tau$-quasinormal in $G$.

Proof. Let $Q$ be a Sylow $q$-subgroup of $G$ and $\left(p,\left|Q^{G}\right|\right) \neq 1$, where $q$ is a prime distinct from $p$. Since $|N|_{q}=|H N|_{q}$ and $N \cap Q$ is a Sylow $q$-subgroup of $N$, we have that $N \cap Q=H N \cap Q$, i.e., $(H \cap Q)(N \cap Q)=H N \cap Q$. By Lemma 2.3, we have $H Q \cap N Q=(H \cap N) Q$. Thus $H \cap N$ is $\tau$-quasinormal in $G$.

Lemma 2.5. Let $G$ be a group and $p$ a prime dividing $|G|$ with $(|G|, p-1)=1$.

(1) If $G$ has cyclic Sylow p-subgroups, then $G$ is p-nilpotent.

(2) If $G$ is p-supersolvable, then $G$ is p-nilpotent.

(3) If $N$ is a normal subgroup of $G$ with order $p$ and $G / N$ is p-supersolvable, then $G$ is p-nilpotent.

Proof. (1) and (2) are [9, Lemma 2.6]. (3) follows directly from (2).

Lemma 2.6 ([11, Lemmas 2.2]). If a subgroup $H$ of $G$ is $\tau$-quasinormal in $G$ and $H \leq O_{p}(G)$ for some prime $p$, then $H$ is $S$-quasinormal in $G$.

Lemma 2.7 ([14, Corollary 1.1]). Let $H$ be a normal subgroup of a group $G$. If every $G$-chief factor below $F^{*}(H)$ is cyclic, then every $G$-chief factor below $H$ is cyclic.

Lemma 2.8 ([12, Theorem A]). If $H$ is an $S$-permutable p-subgroup of $G$ for some prime $p$, then $N_{G}(H) \geq O^{p}(G)$.

Lemma 2.9. Let $H$ be a normal subgroup of a group $G$. Suppose that for every non-cyclic Sylow subgroup $P$ of $H$, all cyclic subgroups of $P$ of prime order and order 4 without supersolvable supplements in $G$ are $\tau$-supplemented in $G$. Then every $G$-chief factor below $H$ is cyclic.

Proof. In fact, there are some typing errors in [10, Theorem 3 and Corollary 1] and " $S$-supplemented" should be " $\tau$-supplemented".

\section{Proof of Theorem 1.3}

Theorem 3.1. Let $P$ be a Sylow p-subgroup of a group $G$, where $p$ is a prime divisor of $|G|$ with $(|G|, p-1)=1$. If every maximal subgroup of $P$ not having a p-nilpotent supplement in $G$ is $\tau$-supplemented in $G$, then $G$ is p-nilpotent.

Proof. Suppose that the theorem is false and let $G$ be a counterexample of minimal order. 
(1) $O_{p^{\prime}}(G)=1$.

Assume that $R=O_{p^{\prime}}(G) \neq 1$. Then, obviously, $P R / R$ is a Sylow $p$-subgroup of $G / R$. Suppose that $M / R$ is a maximal subgroup of $P R / R$. Then there exists a maximal subgroup $P_{1}$ of $P$ such that $M=P_{1} R$. If $P_{1}$ has a $p$-nilpotent supplement $D$ in $G$, then $M / R$ has a $p$-nilpotent supplement $D R / R$ in $G / R$. If $P_{1}$ is $\tau$ supplemented in $G$, then $M / R$ is $\tau$-supplemented in $G / R$ by Lemma 2.1(3). Hence $G / N$ satisfies the hypothesis of the theorem. The minimal choice of $G$ implies that $G / R$ is $p$-nilpotent and so $G$ is also $p$-nilpotent, a contradiction.

(2) $G$ is solvable.

If every maximal subgroup of $P$ has a $p$-nilpotent supplement in $G$, then $G$ is $p$-nilpotent by Lemma 2.2. Hence there exists a maximal subgroup $V$ of $P$ such that $V$ is $\tau$-supplemented in $G$. Then there is a non-p-nilpotent subgroup $T$ of $G$ such that $G=V T$ and $V \cap T \leq V_{\tau G}$. Since $O_{p^{\prime}}(G)=1$, we have that $p \| Q^{G} \mid$ for every non-trivial Sylow $q$-subgroup $Q$ of $G(p \neq q)$. Hence $V_{\tau G} Q=Q V_{\tau G}$. This shows that $V_{\tau G}$ is $S$-semipermutable in $G$. If $V_{\tau G}=1$, then, by Lemma 2.5(1), $T$ is $p$-nilpotent, a contradiction. Hence $V_{\tau G} \neq 1$. Let $L$ be a minimal normal subgroup of $G$ contained in $\left(V_{\tau G}\right)^{G}$. By virtue of [5, Theorem A], $L$ is solvable. Consequently, $L \leq O_{p}(G)$. By Lemma 2.1(2), it is easy to see that every maximal subgroup of $P / L$ not having a $p$-nilpotent supplement in $G / L$ is $\tau$-supplemented in $G / L$. Hence $G / L$ satisfies the hypothesis of the theorem. The minimal choice of $G$ implies that $G / L$ is $p$-nilpotent. Since $(|G / L|, p-1)=1$, it follows that $G / L$ is solvable by the well-known Feit-Thompson theorem. Consequently, $G$ is solvable.

(3) The final contradiction.

Let $N$ be a minimal normal subgroup of $G$. From steps (1) and (2), $N \leq O_{p}(G)$. Using the same argument as in the proof of step (2), we have $G / N$ is $p$-nilpotent. Since the class of all $p$-nilpotent groups is a saturated formation, $N$ is the unique minimal normal subgroup of $G$ and $\Phi(G)=1$. Consequently, $G$ has a maximal subgroup $M$ such that $N \nsubseteq M$. Obviously, $N \cap M$ is normal in $G$. The minimal normality of $N$ yields that $N \cap M=1$ and so $G / N \cong M$ is $p$-nilpotent.

Let $V$ be an arbitrary maximal subgroup of $P$. Next we shall prove that if $V$ is $\tau$-supplemented in $G$, then $V$ has a $p$-nilpotent supplement in $G$. Assume there is a subgroup $T$ of $G$ such that $G=V T$ and $V \cap T \leq V_{\tau G}$. If $N \cap V_{\tau G} \neq 1$, then, by Lemma 2.4, $N \cap V_{\tau G}$ is $\tau$-quasinormal in $G$. By virtue of Lemma 2.6, $N \cap V_{\tau G}$ is $S$ quasinormal in $G$. Furthermore, $O^{p}(G) \leq N_{G}\left(N \cap V_{\tau G}\right)$ from Lemma 2.8. It follows that $N=\left(N \cap V_{\tau G}\right)^{G}=\left(N \cap V_{\tau G}\right)^{O^{p}(G) P}=\left(N \cap V_{\tau G}\right)^{P} \leq\left(V_{\tau G}\right)^{P} \leq V^{P}=V$. This implies that $G=V M$ and $V$ has the $p$-nilpotent supplement $M$ in $G$. If 
$N \cap V_{\tau G}=1$, then $V \cap T \cap N=1$ and so $|T \cap N|=|T \cap N: V \cap T \cap N|=$ $|(T \cap N) V: V| \leq|N V: V| \leq|P: V| \leq p$. Since $T / T \cap N \cong T N / N \leq G / N$, we have that $T / T \cap N$ is $p$-nilpotent. In view of Lemma 2.5(3), $T$ is $p$-nilpotent as desired.

Now we have that every maximal subgroup of $P$ has a $p$-nilpotent supplement in $G$. Applying Lemma 2.2, $G$ is $p$-nilpotent, a contradiction.

Corollary 3.2. Let $P$ be a Sylow p-subgroup of a group $G$, where $p$ is a prime divisor of $|G|$ with $(|G|, p-1)=1$. If every maximal subgroup of $P$ not having a p-supersolvable supplement in $G$ is $\tau$-supplemented in $G$, then $G$ is p-nilpotent.

Proof. It follows directly from Lemma 2.5(2) and Theorem 3.1.

Corollary 3.3. Let $P$ be a Sylow p-subgroup of a group $G$, where $p$ is the smallest prime dividing $|G|$. If every maximal subgroup of $P$ not having a supersolvable supplement in $G$ is $\tau$-supplemented in $G$, then $G$ is p-nilpotent.

Theorem 3.4. Let $H$ be a normal subgroup of a group $G$. Suppose that for every non-cyclic Sylow subgroup $P$ of $H$, each maximal subgroup of $P$ not having a supersolvable supplement in $G$ is $\tau$-supplemented in $G$. Then every $G$-chief factor below $H$ is cyclic.

Proof. Suppose that this theorem is false and let $(G, H)$ be a counterexample for which $|G|+|H|$ is minimal. By Corollary 3.3 and Lemma 2.5, $H$ is $p$-nilpotent, where $p$ is the smallest prime dividing $|H|$. Let $V$ be a normal $p$-complement of $H$. Then $V$ is normal in $G$ since it is characteristic in $H$. Moreover, by Lemma 2.1, the hypothesis holds for $(G / V, H / V)$. Hence in the case when $V \neq 1$, we have every $G / V$-chief factor below $H / V$ is cyclic by the choice of $(G, H)$. It is clear that $(G, V)$ also satisfies the hypothesis. Hence each $G$-chief factor below $V$ is cyclic again by the choice of $(G, H)$. It follows that every $G$-chief factor below $H$ is cyclic, a contradiction. Hence $V=1$, which implies that $H$ is a $p$-group. In view of [10, Theorem 4], every $G$-chief factor below $H$ is cyclic. This contradiction completes the proof.

Proof of Theorem 1.3. Applying Theorem 3.4 and Lemma 2.9, every G-chief factor below $X$ is cyclic. Since $F^{*}(H) \leq X$, we have that every $G$-chief factor below $F^{*}(H)$ is cyclic. Consequently, every $G$-chief factor below $H$ is cyclic by virtue of Lemma 2.7 . 


\section{Applications}

Theorem 4.1. Let $p$ be a prime divisor of $|G|$ with $(|G|, p-1)=1$ and $H$ a normal subgroup of $G$ such that $G / H$ is p-nilpotent. If there exists a Sylow p-subgroup $P$ of $H$ such that every maximal subgroup of $P$ not having a p-nilpotent supplement in $G$ is $\tau$-supplemented in $G$, then $G$ is p-nilpotent.

Proof. By Lemma 2.1(1), every maximal subgroup of $P$ not having a $p$-nilpotent supplement in $H$ is $\tau$-supplemented in $H$. Applying Theorem 3.1, $H$ is $p$-nilpotent. Let $V$ be the normal $p$-complement of $H$. Then $V$ is normal in $G$ since it is characteristic in $H$. If $V \neq 1$, then it is easy to see that $G / V$ satisfies the hypothesis of the theorem by virtue of Lemma 2.1(3). Hence $G / V$ is $p$-nilpotent by induction. It follows that $G$ is $p$-nilpotent. Next we assume that $V=1$, i.e. $H=P$. Since $G / P$ is $p$-nilpotent, we may let $K / P$ be the normal Hall $p^{\prime}$-subgroup of $G / P$. By Schur-Zassenhaus Theorem, $K=P K_{p^{\prime}}$. Applying Lemma 2.1(1) and Theorem 3.1 again, $K=P \times K_{p^{\prime}}$. Obviously, $K_{p^{\prime}}$ is also a normal $p$-complement of $G$ and so $G$ is $p$-nilpotent.

A subgroup $H$ of $G$ is said to be $c$-supplemented in $G$ if there exists a subgroup $K$ of $G$ such that $G=H K$ and $H \cap K \leq H_{G}$, where $H_{G}$ is the largest normal subgroup of $G$ contained in $H$ (see [1]).

Corollary 4.2 ([3, Theorem 3.4]). Let $P$ be a Sylow p-subgroup of $G$, where $p$ is the smallest prime dividing $|G|$. If all maximal subgroups of $P$ are c-supplemented in $G$, then $G$ is p-nilpotent.

Corollary 4.3 ([15, Theorem 3.3]). Let $p$ be the smallest prime divisor of $|G|$ and $P$ a Sylow p-subgroup of $G$. If every maximal subgroup of $P$ is $S$-semipermutable in $G$, then $G$ is p-nilpotent.

Theorem 4.4. Let $\mathfrak{F}$ be a saturated formation containing the class of all supersoluble groups $\mathfrak{U}$ and $H$ a normal subgroup of a group $G$ such that $G / H \in \mathfrak{F}$. Let $X$ be a normal subgroup of $G$ with $F^{*}(H) \leq X \leq H$. Suppose that for every non-cyclic Sylow subgroup $P$ of $X$, either all maximal subgroups of $P$ lacking supersolvable supplements in $G$ or all cyclic subgroups of $P$ of prime order and order 4 without supersolvable supplements in $G$ are $\tau$-supplemented in $G$. Then $G \in \mathfrak{F}$.

Proof. Applying Theorem 1.3, every $G$-chief factor below $H$ is cyclic. By [2, IV, Proposition 3.11], the chief factors of $H$ are $f$-central. And since $G / H \in \mathfrak{F}$, the chief factors of $G / H$ are $f$-central. Thus all chief factors of $G$ are $f$-central, i.e. $G \in \mathfrak{F}$. 
Corollary 4.5 ([3, Theorem 4.2]). Let $\mathfrak{F}$ be a saturated formation containing $\mathfrak{U}$ and $H$ a normal subgroup of a group $G$ such that $G / H \in \mathfrak{F}$. If every maximal subgroup of any Sylow subgroup of $H$ is c-supplemented in $G$, then $G \in \mathfrak{F}$.

Corollary 4.6 ([16, Theorem 1.1]). Let $\mathfrak{F}$ be a saturated formation containing $\mathfrak{U}$ and $H$ a normal subgroup of a group $G$ such that $G / H \in \mathfrak{F}$. If all maximal subgroups of any Sylow subgroup of $F^{*}(H)$ are c-supplemented in $G$, then $G \in \mathfrak{F}$.

Corollary 4.7 ([16, Theorem 1.2]). Let $\mathfrak{F}$ be a saturated formation containing $\mathfrak{U}$ and $H$ a normal subgroup of a group $G$ such that $G / H \in \mathfrak{F}$. If all minimal subgroups and all cyclic subgroups with order 4 of $F^{*}(H)$ are c-supplemented in $G$, then $G \in \mathfrak{F}$.

Acknowledgement. The author would like to thank the referee for the valuable suggestions and comments.

\section{References}

[1] A. Ballester-Bolinches, Y. Wang and G. Xiuyun, c-supplemented subgroups of finite groups, Glasg. Math. J., 42(3) (2000), 383-389.

[2] K. Doerk and T. Hawkes, Finite Soluble Groups, De Gruyter Expositions in Mathematics, 4, Walter de Gruyter \& Co., Berlin, 1992.

[3] X. Guo and K. P. Shum, Finite p-nilpotent groups with some subgroups csupplemented, J. Aust. Math. Soc., 78(3) (2005), 429-439.

[4] B. Huppert and N. Blackburn, Finite Groups III, Fundamental Principles of Mathematical Sciences, 243, Springer-Verlag, Berlin-New York, 1982.

[5] I. M. Isaacs, Semipermutable $\pi$-subgroups, Arch. Math. (Basel), 102(1) (2014), $1-6$.

[6] O. H. Kegel, Sylow-gruppen und subnormalteiler endlicher gruppen, Math. Z., 78(1) (1962), 205-221.

[7] C. Li, Finite groups with some primary subgroups SS-quasinormally embedded, Indian J. Pure Appl. Math., 42(5) (2011), 291-306.

[8] S. Li, Z. Shen, J. Liu and X. Liu, The influence of SS-quasinormality of some subgroups on the structure of finite groups, J. Algebra, 319(10) (2008), 42754287.

[9] C. Li, N. Yang and N. Tang, Some new characterisations of finite p-supersoluble groups, Bull. Aust. Math. Soc., 89(3) (2014), 514-521.

[10] C. Li, X. Zhang and X. Yi, On $\tau$-supplemented subgroups of finite groups, Miskolc Math. Notes, 14(3) (2013), 997-1008. 
[11] V. O. Lukyanenko and A. N. Skiba, On weakly $\tau$-quasinormal subgroups of finite groups, Acta Math. Hungar., 125(3) (2009), 237-248.

[12] P. Schmidt, Subgroups permutable with all Sylow subgroups, J. Algebra, 207(1) (1998), 285-293.

[13] A. N. Skiba, On weakly s-permutable subgroups of finite groups, J. Algebra, 315(1) (2007), 192-209.

[14] A. N. Skiba, On two questions of L. A. Shemetkov concerning hypercyclically embedded subgroups of finite groups, J. Group Theory, 13(6) (2010), 841-850.

[15] L. Wang and Y. Wang, On s-semipermutable maximal and minimal subgroups of Sylow p-subgroups of finite groups, Comm. Algebra, 34(1) (2006), 143-149.

[16] H. Wei, Y. Wang and Y. Li, On c-supplemented maximal and minimal subgroups of Sylow subgroups of finite groups, Proc. Amer. Math. Soc., 132(8) (2004), 2197-2204.

[17] Q. Yan, X. Bao and Z. Shen, Finite groups with SS-supplement, Monatsh. Math., 184(2) (2017), 325-333.

\section{Changwen Li}

School of Mathematics and Statistics

Jiangsu Normal University

221116 Xuzhou, China

e-mail: lcw2000@126.com 